\title{
ANOTACIONES SOBRE EUFEMISMOS Y FALSOS AMIGOS EN EL ALEMÁN ECONÓMICO ACTUAL: APROXIMACIÓN TEÓRICA Y TRADUCCIÓN AL ESPAÑOL
}

\author{
Alfonso Corbacho Sánchez \\ Universidad de Extremadura
}

\begin{abstract}
Unlike other Languages for Specific Purposes such as legal or scientific-technical, business German has not always deserved the same attention from researchers. Yet, few will doubt that the analysis and teaching of business German is becoming more and more important in the Spanish universities. In this sense, the aim of the research presented here is to show a theoretical approach and a lexical-semantic analysis of metaphors, euphemisms and false friends in German for business language and its translation into Spanish. The corpus used consists of a large number of papers that come from current business magazines and newspapers.
\end{abstract}

\section{INTRODUCCIÓN}

El creciente interés científico por los lenguajes especializados provoca, hoy en día, continuos trabajos de investigación sobre este campo del conocimiento. No obstante, llama la atención el hecho de que esta progresiva atención investigadora que se presta a los lenguajes especializados se ha centrado, tradicionalmente, más en la parcela científicotécnica y jurídica que en el ámbito de la economía.

En la actualidad, el lenguaje económico está comenzando a recuperar a pasos agigantados todo el terreno perdido. En sólo unos años, el léxico económico se ha convertido en moneda de cambio de todos los públicos ${ }^{1}$. Si antes quedaba restringido a los expertos, ahora cualquier profano en la materia es capaz de reconocer y entender algún que otro término de contenido especializado procedente del mundo de la economía que por su frecuencia de uso ha acabado por implantarse en el lenguaje general. Todo ello se debe en gran parte a los medios de comunicación pero, sobre todo, al cuantioso número de publicaciones periódicas ${ }^{2}$ dedicadas a la economía.

\footnotetext{
${ }^{1}$ Así se expresa G. Scholtes-Schmid (1986: 35), para quien la relación del lenguaje económico con la vida cotidiana supera los vínculos que pueden existir con otras áreas del conocimiento. De este modo, afirma que "der Bereich der Wirtschaft in all seinen Ausprägungen in viel stärkerem Maß mit dem Alltagsleben verflochten ist als andere Bereiche".

${ }^{2}$ Véase en las referencias bibliográficas el suculento número de revistas y periódicos en lengua alemana pertenecientes al ámbito de la economía que, por otra parte, configura el material de trabajo de la presente investigación a la hora de extraer el léxico para su posterior análisis.
} 
En esta línea, el objetivo vertebrador del presente trabajo consiste en mostrar una aproximación teórica y un análisis léxico-semántico de los eufemismos y los falsos amigos en el alemán económico junto a su traducción al español. Tras la confección del corpus que incluye una serie de textos de carácter económico, el estudio implica dos fases bien delimitadas:

- $\quad$ un primer rastreo de los ejemplos susceptibles de valor evaluativo

- el análisis léxico-semántico propiamente dicho

\section{EL CORPUS}

En relación a los textos, se ha seleccionado documentación específica de temas económicos. Los textos del registro económico que conforman el corpus son en su totalidad documentos procedentes de revistas y periódicos alemanes con una representatividad aceptable en lo que concierne al ámbito de la economía. Consideramos dichos textos como representativos de la disciplina económica en lengua alemana por el carácter reciente de las publicaciones -toda la prensa especializada que hemos seleccionado data del 2002-; por el elevado parámetro de impacto con que cuentan los periódicos y revistas seleccionados dentro del área al que se adscriben; y por tratarse de unas publicaciones periódicas cuyo contenido está constituido, como ya hemos indicado, esencialmente por cuestiones económicas ${ }^{3}$.

Así pues, tras el examen del material de nuestra selección, el corpus que constituye la base empírica de nuestro trabajo consiste en 17 artículos del campo de la economía. La muestra se divide en 4 artículos de periódicos y 13 de revistas de extensión variable, es decir, 17 títulos extraídos de 11 publicaciones diferentes ${ }^{4}$, que pueden considerarse lo suficientemente representativos y relevantes del área de conocimiento al que pertenecen como para que los datos obtenidos resulten fiables.

\section{EUFEMISMOS EN EL ALEMÁN DE LA ECONOMÍA}

Un tema tabú en la sociedad genera frecuentemente una expresión lingüística negativa y desagradable. Pues bien, el eufemismo es una expresión atenuada con cierto decoro que sustituye esa noción o idea impopular transmitiendo el mismo contenido y eliminando todo aspecto que pueda considerarse como ofensivo por tratarse de palabras socialmente más aceptables y desprovistas de connotaciones negativas. H. Glück (1993: 176) sintetiza la explicación precedente y escribe al respecto lo siguiente:

\footnotetext{
${ }^{3}$ Somos conscientes de que el lenguaje de la economía no sólo proviene de la prensa especializada, a pesar de ello no entraremos en la eterna diatriba sobre cuáles deberían ser las cuestiones económicas fundamentales que habría que incluir en un trabajo de estas características. Interesante sería, sin duda alguna, realizar en otro estudio un análisis léxico-semántico de textos procedentes de la correspondencia comercial, declaración de hacienda, contratos comerciales, etc. (Hundt 1998: 1297). En definitiva, son cada vez más las publicaciones periódicas de contenido exclusivamente económico con tiradas considerables. Prueba fehaciente de ello son las revistas y los periódicos que hemos seleccionado.

${ }^{4}$ Véanse todas las publicaciones de carácter económico consignadas en el apartado final de las referencias bibliográficas.
} 
"durch gesellschaftliche, ideologische oder religiöse Konventionen verursachte beschönigende, 'verhüllende' Ausdrucksweise".

También en el lenguaje económico-comercial se divisan algunas manifestaciones eufemísticas ${ }^{5}$ y en otros terrenos muy afines como la política y la propaganda comercial (Lewandowski 1984: 279; Glück 1993: 176).

Ciertamente, es notoria la necesidad de recurrir a expresiones eufemísticas para tratar de paliar los efectos adversos que produce la mención de realidades desagradables como, en conexión con la economía, el desempleo o, al margen de temas económicos, las referencias a la tercera edad.

Mostramos a continuación algunas expresiones halladas en nuestro corpus que constituyen claros ejemplos de lo que se entiende por eufemismo:

a) Für den Euro gibt es weiterhin Risiken, doch überwiegen derzeit wegen der DollarSchwäche die Chancen auf eine Kurserholung. (Börse online: 58-59)

El ejemplo expuesto aglutina dos fenómenos lingüísticos susceptibles de comentario. Por una parte, la carga eufemística de Schwäche ("debilidad") suavizando la situación negativa por la que atraviesa el dólar y, por otra parte, el sentido metafórico que implica el mismo término al atribuir rasgos humanos a la moneda americana.

b) Ebbe in den Rentenkassen. (Die Telebörse: 71)

El vocablo Ebbe ("marea baja") vuelve a ser una muestra en la que confluyen tanto matices metafóricos como eufemísticos cuando se refiere a la falta de fondos para cubrir las pensiones.

c) Käme es auf Grund zu starker Lohnsteigerungen zu einem weiteren Stellenabbau, würde das die Konsumneigung der Verbraucher belasten. (Euro am Sonntag: 8)

Stellenabbau ("despido de personal") se transforma en un eufemismo por disfrazar con discreción el despido y sustituir el término más usual Entlassung (“despido").

d) Dresdner prüft Job-Abbau im Investmentbanking. (Handelsblatt: 30)

Eufemismo idéntico a Stellenabbau con la salvedad de que en esta ocasión se trata de un híbrido compuesto por el anglicismo Job y el término autóctono Abbau.

e) Für einen Wechsel können eine Beitragsersparnis und ein besserer Versicherungsschutz sprechen. (Finanz€n: 93-95)

Otro caso más centra su atención en el empleo eufemístico de Beitrag ("contribución" o "cuota"), uno de los términos que se utilizan para encubrir el término Steuern ("impuestos").

\footnotetext{
${ }^{5}$ Sobre el estudio de los eufemismos en la lengua alemana es de extraordinario interés el trabajo de Sigrid Luchtenberg (1985), en especial el apartado que entronca con nuestro tema «Euphemismen im ökonomischen Sprachbereich» (págs. 58-76). Consúltese también el estudio de Leinfellner (1971).
} 
f) Zudem sind viele Konzerne noch mit relativ üppigen Investitionsplänen in das Rezessionsjahr 2001 gestartet. (Handelsblatt: 15)

El anglicismo Rezession ("recesión") ya se ha extendido hasta tales niveles, que en la comunicación especializada y no tan especializada se ha transformado en un término asiduo en los quehaceres cotidianos de los economistas. Sin embargo, por hacer referencia a una disminución del producto nacional bruto seguida de una reducción del empleo y de la producción camufla una situación claramente negativa.

g) Bei den zehn führenden Automobilherstellern erhöhte sich die Finanzverschuldung seit Ende 1999 und rund 100 Mrd. \$, während das Geschäft nahezu stagnierte... (Handelsblatt: 15)

El verbo stagnieren ("estancar") suaviza lo que en el fondo es, a todas luces, una paralización comercial. Para dicho propósito recurre a un eufemismo metafórico en el momento que alude a fenómenos de la naturaleza por el estancamiento de, por ejemplo, un río, un arroyo o bien del agua en general, trasladando esta parada a todo tipo de operaciones comerciales.

A tenor de lo expuesto, podemos afirmar que los eufemismos son fenómenos lingüísticos que, a pesar de mostrarse con mayor moderación frente a unidades léxicas más usuales en este tipo de textos -anglicismos, galicismos, acrónimos, etc.-, sí pueden ser catalogados como una característica específica del lenguaje económico.

\section{FALSOS AMIGOS ESPECÍfICOS DEL ALEMÁN ECONÓMICO}

En este apartado procederemos a desenredar la confusión que pueden ocasionar algunos casos de falsos amigos asentados en nuestro corpus de textos. Una primera elucidación del concepto de falsos amigos (Glück, 1993: 182) apunta, en líneas generales, que se trata de

“...morphologische und idiomatische Entsprechungen zwischen zwei Sprachen, wenn sich zwei Wörter oder Wendungen scheinbar entsprechen, aber unterschiedliche Referenzbereiche haben".

Pues bien, los falsos amigos aluden a vocablos de dos lenguas diferentes que coinciden en su apariencia externa, o cuando menos son bastante similares al pasar de una lengua a otra, pero con discrepancias en el terreno de la semántica, pues corresponden a dos realidades diferentes.

También son muy aclaratorias las palabras del profesor G. Wotjak (1996: 125-126) con las que glosa que los falsos amigos:

...son unidades léxicas (UL) que se caracterizan por coincidencias totales o parciales en lo formal, o sea, en el plano de la expresión y en lo semántico comunicativo, o sea, en el plano del contenido, y que se destacan al mismo tiempo por divergencias más o menos considerables en uno de estos dos planos constitutivos para cualquier UL como signo lingüístico bilateral.

En esta dirección, el autor distingue entre falsos amigos semánticos y falsos amigos formales o estructurales, pero son los denominados semánticos los que albergan un mayor 
grado de dificultad. En referencia a los falsos amigos semánticos, los estudiosos G. Wotjak y U. Herrmann (1993: 6) señalan:

"Diese erweisen sich oft als trügerisch, weil sie eine Bedeutungsgleichheit suggerieren, die im Laufe der Sprachentwicklung wie auch im z.T. mehrfach vermittelten Entlehnungsproze $\beta$ verlorengegangen ist".

Asimismo, en el trabajo Vergleichende Grammatik Spanisch-Deutsch de los autores Cartagena y Gauger (1989: 581 y sigs.) los falsos amigos quedan clasificados en seis tipos diferentes:

1) “ähnliche materielle Form - verschiedene Bedeutung”. En el sentido literal del término, se trata del falso amigo por excelencia, es decir, estructura formal parecida con significado totalmente diferente.

2) "Hier stimmt eine der beiden oder mehrere Bedeutungen überein mit der des materiall ähnlichen Worts der anderen Sprache". Se produce cuando a una palabra le corresponde un significado de entre dos o más términos posibles de los cuales uno presenta una estructura externa muy parecida. Uno de los ejemplos que citan los autores es el término Tourismus, que en la lengua española puede significar tanto el flujo de turistas como un automóvil determinado.

3) "Hier sind in der einen Sprache zwei oder mehrere Bedeutungen auf zwei oder mehr materiell verschiedene Wörter verteilt, während sie in der anderen durch ein einziges Wort ausgedrückt werden; ...". Este tercer tipo es idéntico al anterior, si exceptuamos que en este caso no existe ningún parecido en la estructura formal. Sin embargo, insistimos en que a una palabra de una lengua le pueden corresponder dos o más palabras de otra lengua distinta.

4) "es besteht die Tendenz, die materielle Form des Worts der einen auf das entsprechende, materiell sehr ähnliche, der anderen Sprache zu übertragen". En el cuarto ejemplo tiene lugar una interferencia formal y no semántica como hemos podido apreciar en los tres tipos anteriores. Aquí, se produce un traspaso de la forma externa de una palabra en una lengua a otra lengua con los posibles errores morfológicos y ortográficos que ello pueda acarrear.

5) “... durch die große materielle Ähnlichkeit bedingt, das Genus des Worts der einen Sprache auf das entsprechende der anderen zu übertragen, ...". Este quinto tipo de falso amigo se apoya en el género de las palabras. La coincidencia formal de dos vocablos puede traer consigo el traspaso del género y el consecuente error, cuando el término de una lengua muestre un género distinto en otra.

6) “Auch hier besteht die Gefahr,(...), materieller Übertragung. Nur ist in diesem Fall das Wort der anderen Sprache materiell völlig verschieden”. Este último tipo es muy similar al anterior, con una salvedad, ahora el traspaso de género se produce con palabras sin ningún parecido en su forma externa.

Otro detalle a considerar en el trabajo de estos autores versa sobre su postura ante el componente etimológico (Cartagena/Gauger 1989: 582) de los falsos amigos. Cartagena y Gauger insisten en una definición estrictamente sincrónica del término, pese a que una gran mayoría presenta rasgos etimológicos comunes, dado que la trayectoria histórica de dos 
palabras es culturalmente distinta $\mathrm{y}$, por tanto, irrelevante al objeto de poder desentrañar el significado de las mismas.

Por el contrario, el lingüista Gerd Wotjak, tanto en lo relativo a los falsos amigos como a los verdaderos amigos, sí concede la debida importancia al aspecto etimológico, resaltando que en la mayoría de los casos

"auf ein gemeinsames Etymon zurückgehen" (Wotjak 1990: 18) ${ }^{6}$. Si bien, en un principio, perfila "dass formale Koinzidenz nicht notwendig auf ein gemeinsames Etymon zurückzuführen ist" (Wotjak 2002: 93) ${ }^{7}$,

más adelante, matiza su punto de vista y llega a la coherente conclusión de que:

Die überwiegende Mehrzahl von formalen Identitäten im Sprachenpaar lässt sich dagegen sehr wohl auf ein gemeinsames Etymon zurückführen, wobei es sich um Entlehnungen aus einer gemeinsamen Drittsprache, in der Vergangenheit und ausgewählten Wissensbereichen vor allem aus dem Latein, neuerdings vor allem aus dem Englischen, bzw. auch aus jeweils der anderen Sprache handelt. (Wotjak 2002: 100)

Tras estas sucintas consideraciones teóricas, podemos fijar una primera conclusión. El estudio de los postulados teóricos verifica que, a pesar de las enormes diferencias estructurales entre las lenguas alemana y española, sí podemos dar fe de la existencia de este fenómeno lexicológico. Sirva la extraordinaria recopilación llevada a cabo por Wotjak (1993) titulada Typische Fehler Spanisch como testimonio de la suculenta relación de falsos amigos que existen entre ambas lenguas. Por consiguiente, será la tarea, de aquí en adelante, comprobar la existencia de algunas muestras en el lenguaje especializado de la economía.

Sin embargo, no es éste un campo profuso en ejemplos, dado que el alemán y el español no son lenguas de estrechos lazos lingüísticos. A pesar de todo ello, hemos podido reunir una serie de falsos amigos muy significativos para nuestra investigación por su pertenencia al lenguaje de la economía.

En definitiva, centraremos toda nuestra atención en descubrir y someter a examen el grupo de los falsos amigos más relevantes por su variedad y por el peso específico que tienen en el estudio de las lenguas extranjeras. Sin duda alguna, con ello nos referimos a los falsos amigos semánticos ${ }^{8}$.

A continuación, procederemos a exponer varios ejemplos de falsos amigos junto a un breve comentario sobre los mismos, destacando el fuerte vínculo que une las muestras al lenguaje de la economía:

\footnotetext{
${ }^{6}$ En esta misma línea, consúltense las posturas -compartidas por Wotjak- de Kühnel (1974: 115) y Gottlieb (1986: 105 y sig.).

${ }^{7}$ Una postura más diplomática proviene de M. Wandruszka (1977: 59) al indicar que una serie de falsos amigos sí nos pueden remitir al criterio etimológico, mientras que otros no respetan el principio de la etimología.

${ }^{8}$ Con independencia de los autores y sus propuestas clasificatorias, Wotjak (1993) los denomina falsos amigos semánticos, I. Doval (1998) los cataloga como totale falsche Freunde y Cartagena/Gauger (1989) los describen como ähnliche materielle Form - verschiedene Bedeutung. En definitiva, todas las designaciones aluden a los falsos amigos que a más equívocos conducen.
} 
Auch Rohstoffe und Devisen gehören zum Anlagehorizont. (Aktien \& Co.: 60-61)

Citemos como primer ejemplo Devisen que traducido es "divisa", moneda de un país, frente a la traducción errónea de "devisa" en español.

Das hätte die Ölimport-Rechnung -und damit die Leistungsbilanz- spürbar entlasten können. (Börse online: 58-59)

El término alemán Import se refiere a la "importación", que habitualmente coincide en español frente a una segunda acepción que alude al importe.

a) Hohe Flexibilität, da der Gründer je nach Bedarf Geld für seinen persönlichen Lebensunterhalt aus seiner Firma herausziehen kann. (Capital: 68)

Incorporamos el vocablo Firma como falso amigo, pese a que nos permitimos señalar algunas observaciones al respecto. En el contexto que nos ocupa Firma se aplica a empresas, que suele ser el significado más utilizado en alemán, mientras que en español firma se refiere mayoritariamente al acto de firmar cualquier documento escrito y no a una empresa determinada. En suma, el término queda recogido en la lengua española con ambas acepciones, pese a que, por regla general, la primera de ellas muestra un ligero predominio sobre la segunda.

...da bei diesen Unternehmen der Unterschied zwischen den Buchwerten in der Bilanz und den tatsächlichen Marktwerten in der Regel relativ gering ist. (Der Aktionär: 88-89)

Otro ejemplo típico es Bilanz, que además de "balance" se usa de manera equivocada para designar una "balanza".

Sollte die Wirtschaftsflaute anhalten, ... (Die Telebörse: 71)

Otro ejemplo muy escurridizo es Wirtschaftsflaute. La similitud morfológica de la palabra alemana Flaute con el instrumento musical flauta en español está fuera de toda duda. Pues bien, su significado en el lenguaje bursátil no es otro que el de "calma económica" o "estancamiento económico". Como vemos, bastante lejos del enclave musical que puede suponer a priori.

Liegt der Kurs der Aktie dann über dem ermittelten Value, ist die Aktie überbewertet und damit ein Verkauf;... (DMEuro: 88-89)

El vocablo Aktie sólo puede corresponder al término español "acción”, generalmente utilizado en plural "acciones", en alusión a cada una de las partes indivisibles del capital de una sociedad. En ocasiones, la ambivalencia de significado de un vocablo en la traducción nos puede conducir a versiones erróneas.

...können Anleger, die heute investieren, mit Sicht auf fünf bis zehn Jahre eigentlich mit noch höheren Renditen rechnen. (FinanzEn: 54-55)

Un ejemplo más, utilizado en centenares de circunstancias por el colectivo de economistas, es investieren, que en alemán es "invertir" y que en español puede confundirse con "investir". 
Als Anlagevehikel, um vom Wachstum nicht notierter Unternehmen zu profitieren, bieten sich prinzipiell drei Formen an... (Finanz $n$ : 54-55)

Un ejemplo al margen del lenguaje de la economía lo compone el término prinzipiell que, en ocasiones, es traducido por "principalmente" en lugar de "por principio" o "de principio".

Weil sich Fondsmanager Lawrence Kymisis aber nicht ausschließlich auf Wachstumswerte konzentrierte, erreichte der Fonds dennoch bessere Ergebnisse als die Konkurrenten. (Geldidee: 26)

Als Frühindikator konkurriert die ZEW-Konjunkturerwartung dabei mit dem ifoGeschäftsklimaindex. (Die Telebörse: 65)

Otro tipo de discrepancia está en el uso de Konkurrenten que nos conduce directamente a "concurrente", pero no es ése su significado, sino el de "competidores". Lo mismo sucede con el verbo konkurrieren que viene a significar "competir" o "hacer la competencia" en lugar de lo que sugiere ("concurrir").

...wobei der Anstieg auf stärkere Schwankungen der Risikoprämie und der Prämie für höhere Liquidität ebenso zurückgeht,... (Financial Times: 21)

Un ejemplo más es el término Prämie, que en un contexto económico-empresarial se traduce al español por "prima" y no en el sentido de "premio".

Die Berichtssaison der Unternehmen war dominiert von Meldungen aus den Technologieund Telekombranchen. (Financial Times: 22)

Otro falso amigo lo constituye el vocablo Saison que se utiliza como "época del año", "estación" o "temporada", mientras que el español nos puede confundir con el término "sesión”.

\section{CONSIDERACIONES FINALES}

A la hora de hacer un balance final deseamos hacer constancia de un apunte de trascendental importancia que gira en torno a la procedencia del material lingüístico seleccionado para nuestro análisis. Las revistas de economía, que configuran la mayor parte de nuestro material, y los periódicos podrían plantear el problema de que no estamos sometiendo a estudio el lenguaje económico sino el lenguaje periodístico. No hemos entrado ni vamos a entrar a examinar el grado de especialización de los diferentes textos seleccionados. La única afirmación seria que podemos alegar al respecto es que la comprensión de un buen número de artículos no siempre está al alcance del lector no especialista. Dichos artículos combinan, entre otros recursos léxico-semánticos, siglas, acrónimos, neologismos y palabras anglosajonas -estas últimas, en muchos casos, sin equivalente en alemán- con tan alta densidad que resulta improbable que puedan ser tachados de textos no especializados. Bajo esta perspectiva, tampoco se encuentran diccionarios que puedan esclarecer algunas dudas halladas en los artículos citados, justificando la confusión que puede crear el nacimiento reciente de ciertas realidades lingüísticas. En efecto, es utópico creer que todo lector no especializado se dedique a 
descifrar y estudiar el último invento bursátil que aparece en algunos de estos escritos, en muchas ocasiones, crípticos.

Una apreciación más, tal vez la más determinante, concerniente al grado de especialización de los textos seleccionados se basa en la habitual diferencia que existe en los artículos de información económica que proceden de un periódico de gran divulgación con respecto a la prensa especializada -como es nuestro caso- del sector de la economía. No sólo es una diferencia cuantitativa, sino también cualitativa, sobre todo, en la presentación de los hechos.

Por otro lado, es de justicia advertir que los resultados del análisis léxico tienen una limitación relativa en cuanto a la extrapolación de los mismos, dado que no estamos ante una representación textual de todas las ramificaciones que pueden derivarse del campo de la economía y máxime cuando los textos proceden en su totalidad de revistas y periódicos del citado campo del saber, excluyéndose monografías, manuales y demás estudios. La naturaleza descriptiva de esta contribución nos impuso una serie de medidas restrictivas, por lo que se realizó la selección de textos atendiendo a unos criterios que se estimaron racionales y objetivos. Por consiguiente, estas matizaciones tienen como consecuencia directa que la yuxtaposición de los resultados no pueda ser extrapolable en todos sus casos a géneros diferentes al analizado, aunque sí pueden servir de orientación y aproximación en cuanto al uso de determinadas estructuras lingüísticas. De todos modos, creemos que todo ello no resta validez a nuestro análisis ni a los resultados obtenidos $\mathrm{y}$, por otro lado, puede suponer un paso más para comprobar en estudios futuros dedicados a otras muestras textuales diferentes, propias del entorno económico, si se cumplen los resultados aquí expuestos.

Para cerrar este apunte final, no podemos negar que, al margen de la inestimable ayuda que nos ha concedido la bibliografía, es el trabajo experimental el pilar básico que sustenta las principales argumentaciones que hemos expuesto en nuestra contribución. Y en este sentido, a pesar del interés que siempre han despertado estos temas, reincidimos en que respecto al análisis léxico en el marco de la economía aún existe una carencia considerable de estudios lingüísticos.

\section{REFERENCIAS BIBLIOGRÁFICAS}

CARTAGENA, N./ GAUGER, H.-M., Vergleichende Grammatik Spanisch-Deutsch (tomo 2), Mannheim, Dudenverlag, 1989.

DOVAL REIXA, I., "Zu dem Begriff 〈Falsche Freunde〉 und seiner praktischen Anwendung am Sprachenpaar Deutsch-Spanisch", Revista de Filología Alemana 6 (1998), pp. 277-289.

FRAAS, C., "Lexikalisch-semantische Eigenschaften von Fachsprachen”, en Hoffmann, L./ Kalverkämper, H./ Wiegand, H.E. (eds.), Fachsprachen/ Languages for Special Purposes. Ein internationales Handbuch zur Fachsprachenforschung und Terminologiewissenschaft (tomo 1), Berlín / Nueva York, Walter de Gruyter, 1998, pp. 428-438.

GLÜCK, H. (ed.), Metzler Lexikon Sprache, Stuttgart, Metzler, 1993. 
GOTTLIEB, K. H., "Grundprinzipien eines Wörterbuchs der Falschen Freunde des Übersetzers. Ein Beitrag zur praktischen Lexikographie», Germanistische Linguistik 3-6 (1986), pp. 103-134.

HEBEL, F., "Sprache der Wirtschaft", Der Deutschunterricht 4 (1969), pp. 58-72.

HUNDT, M., "Neuere institutionelle und wissenschaftliche Wirtschaftsfachsprachen", en Hoffmann, L./ Kalverkämper, H. /Wiegand, H.E. (eds.), Fachsprachen/Languages for Special Purposes. Ein internationales Handbuch zur Fachsprachenforschung und Terminologiewissenschaft (tomo 1), Berlin / Nueva York: Walter de Gruyter, 1998, pp. 1296-1304.

KÜHNEL, H., "Die französischen faux amis im deutschen Wortschatz", Deutsch als Fremdsprache, 2 (1974), pp. 115-117.

LEINFELlNER, E., Der Euphemismus in der politischen Sprache, Berlín, Duncker \& Humblot, 1971.

LEWANDOWSKI, T., Linguistisches Wörterbuch (3 tomos), Heidelberg, Quelle \& Meyer, 1984. (4 $4^{\mathrm{a}}$ ed.)

LUCHTENBERG, J., Euphemismen im heutigen Deutsch, Francfort del Meno, Peter Lang, 1985.

MÖHN, D., "Fach- und Gemeinsprache. Zur Emanzipation und Isolation der Sprache", en Hahn, W. von (ed.), Fachsprachen, Darmstadt, Wissenschaftliche Buchgesellschaft, 1981, pp. 172-217.

SCHOLTES-SCHMID, G., "Die Beschreibung von Wirtschaftsfachsprachen", Fachsprache 1-2 (1986), pp. 35-44.

WANDRUSZKA, M., "Falsche Freunde. Ein linguistisches Problem und seine Lösung»", Festgabe für Julius Wilhelm zum 80. Geburtstag. Zeitschrift für französische Sprache und Literatur (Beiheft, Neue Folge 5), 1977, pp. 53-77.

WOTJAK, G., "Falsche und echte Freunde des Übersetzers. Einige Überlegungen im Spannungsfeld von interlingualen Kongruenzen und Äquivalenzen", Comunicaciones Germánicas 17 (1990), pp. 17-31.

WOTJAK, G./HERRMANN, U., Typische Fehler Spanisch, Berlín, Langenscheidt, 1993. $\left(3^{\mathrm{a}}\right.$ ed. $)$

WOTJAK, G., "Divergencias y congruencias en el léxico entre el español y el alemán. Los llamados falsos amigos del traductor", Sendebar 7 (1996), pp. 125-133.

WOTJAK, G., 'Deutsche Sprach' - schwere Sprach' oder Lexikonvergleich als Sedativum im Sprachenpaar Spanisch-Deutsch", Estudios Filológicos Alemanes 1 (2002), pp. 81-107.

\section{Fuentes secundarias}

Aktien \& Co. Alles über Börsen, Renten, Steuern, Zinsen, nº 14, 2002.

Börse online. Das Anlegermagazin, n 20, 2002.

Capital. Das Wirtschaftsmagazin, $\mathrm{n}^{\circ}$ 10, 2002.

Der Aktionär. Deutschlands grosses Börsenmagazin, nº 20, 2002.

Die Telebörse. Das Anlegermagazin aus Frankfurt, n 18, 2002. 
DMEuro. Das Magazin für Geld und Wirtschaft, $n^{\circ}$ 6, 2002.

Euro am Sonntag, n 20, 2002.

Financial times. Deutschland, $\mathrm{n}^{\circ}$ 99/22, 27-5-2002.

Finanz€n. Börse \& Wirtschaft für Anleger, nº 6, 2002.

Geldidee, $\mathrm{n}^{\circ} 10,2002$.

Handelsblatt. Die Wirtschafts- und Finanzzeitung, nº 99/22, 27-5-2002. 
